\title{
Article
}

\section{Application of Vibration Signal Processing Methods to Detect and Diagnose Wheel Flats in Railway Vehicles}

\author{
JaeSeok Shim ${ }^{1} \mathbb{D}$, GeoYoung Kim ${ }^{2}$, ByungJin $\mathrm{Cho}^{2}$ and JeongSeo Koo ${ }^{1, *}$ \\ 1 Department of Railway Safety Engineering, Seoul National University of Science and Technology, \\ Seoul 01811, Korea; simjaeseok@naver.com \\ 2 Department of Rolling Stock System Engineering, Seoul National University of Science and Technology, \\ Seoul 01811, Korea; mouse1107@daum.net (G.K.); simonbjcho@gmail.com (B.C.) \\ * Correspondence: koojs@seoultech.ac.kr
}

check for updates

Citation: Shim, J.; Kim, G.; Cho, B.; Koo, J. Application of Vibration Signal Processing Methods to Detect and Diagnose Wheel Flats in Railway Vehicles. Appl. Sci. 2021, 11, 2151. https://doi.org/10.3390/app11052151

Academic Editor: César M

A. Vasques

Received: 9 February 2021

Accepted: 24 February 2021

Published: 28 February 2021

Publisher's Note: MDPI stays neutral with regard to jurisdictional claims in published maps and institutional affiliations.

Copyright: (c) 2021 by the authors. Licensee MDPI, Basel, Switzerland. This article is an open access article distributed under the terms and conditions of the Creative Commons Attribution (CC BY) license (https:// creativecommons.org/licenses/by/ $4.0 /)$.

\begin{abstract}
This paper studied two useful vibration signal processing methods for detection and diagnosis of wheel flats. First, the cepstrum analysis method combined with order analysis was applied to the vibration signal to detect periodic responses in the spectrum for a rotating body such as a wheel. In the case of railway vehicles, changes in speed occur while driving. Thus, it is difficult to effectively evaluate the flat signal of the wheel because the time cycle of the flat signal changes frequently. Thus, the order analysis was combined with the existing cepstrum analysis method to consider the changes in train speed. The order analysis changes the domain of the vibration signal from time domain to rotating angular domain to consider the train speed change in the cepstrum analysis. Second, the cross correlation analysis method combined with the order analysis was applied to evaluate the flat signal from the vibration signal well containing the severe field noise produced by the vibrations of the rail irregularities and bogie components. Unlike the cepstrum analysis method, it can find out the wheel flat size because the flat signal linearly increases to the wheel flat. Thus, it is more effective when checking the size of the wheel flat. Finally, the data tested in the Korea Railroad Research Institute were used to confirm that the cepstrum analysis and cross correlation analysis methods are appropriate for not only simulation but also test data.
\end{abstract}

Keywords: fault detection and diagnosis; vibration signal processing technique; wheel flat; cepstrum analysis; cross correlation analysis

\section{Introduction}

A wheel flat is a faulty damage on the wheel tread caused by wheel-rail slipping contacts under train loads due to friction and wear. The wheel flat generates noise and vibration during train operation, and impacts to bearings, gears, motors, etc. Because these phenomena relate to operational life and derailment, anti-skid devices and lubrication have been applied.

In general, small wheel flats are detected by periodical maintenance inspection. However, if there is a large flat with abnormal vibration and noise, they can be found through an unplanned investigation. Unacceptable wheel flats must be repaired in accordance with wheel safety criteria including detailed guidelines on flat size and numbers. Thus, it is necessary that the wheel flats are continuously detected and diagnosed for wheel rectification on time. These days, online monitoring on wheel conditions becomes more important due to high train speed.

For CBM (Condition-Based Maintenance) on flat wheels, various vibration signal processing techniques [1] are applicable. They are very useful for detecting defects in rotating machines, such as gears [2,3] and bearings [4,5]. Duan et al. [6] improved the fault detection rate of a bearing by combining the local mean decomposition and the ratio correction methods. Zheng et al. [7] suggested the spectrum whitening demodulation for a fault diagnosis on axle box bearing. Glowacz [8] suggested a fault diagnosis method 
based on the analysis of thermal images for electric impact drills (EID). However, this is not appropriate for railway maintenance. Liang et al. [9] suggested a time-frequency analysis method for detection and diagnosis of wheel flats. The time-frequency analysis method in the paper includes STFT (Short Time Fourier transform), WVT (Wigner Ville Transform), and WT (Wavelet Trans-form). However, the time-frequency analysis method is difficult to discriminate the defects because it is hard to set a predefined threshold value for a meaningful damage, and actual vibration signals in operation show various frequency bands. Gao et al. [10] suggested a parallelogram method measuring the contact detection of wheel flats on rail. However, this method has a disadvantage that the flat signal is affected by measuring points on rail. Choi and Kim [11] suggested the cepstrum (the minimum distributed cepstrum) as a method which is useful for cyclic defect signal detections at constant velocity, but its effect has not been verified under variable running speed conditions. However, because many accelerations and decelerations take place during real operation of trains, an effective wheel-flat diagnosis algorithm is necessary considering a continuously varying train speed. Wheel flat is one of the main faults of railway vehicle and reduces ride quality too [12]. Thus, this paper studied a vibration signal processing algorithm for early detection of railway wheel flats considering variable train speed. Order and cepstrum analyses were used to effectively detect flats under variable speed conditions for real operation. During this signal processing, zero padding was implemented to facilitate signal detection. The cepstrum analysis was superior in diagnosing the existence of wheel flats with periodic signals, but not their sizes. In addition, to diagnose the size of flats, cross correlation analysis, a good method to diagnose the magnitude of the instantaneous impulse signal, was applied using a periodic signal of wheel flat in terms of repetitive rotation.

Finally, to verify validity of the suggested algorithm, all the analysis procedures were performed and evaluated for wheel flat signals including wheel vibrations in actual field operation.

\section{Cepstrum Analysis for Wheel Flat Detection}

\subsection{Process of the Cepstrum Aanalysis for Harmonic Signal Detection}

In 1963, Bogert et al. [13] proposed a cepstrum signal processing method that can classify and express the first signal and echo signal by the inverse Fourier transformation of logarithm of spectrum signals. After that, several papers $[14,15]$ used it as a signal processing method to detect faults of rotational machines. The cepstrum analysis is effective in detecting periodic components of spectrum signals. It performs Fourier transform, logarithmic calculation, and Fourier inverse transform in order for measured time domain signals [16]. If there is a signal for two wheel flats with time delays $t_{1}$ and $t_{2}$, the signal $x(t)$ can be represented by a convolution of one flat signal with two impulses, as shown in Equation (1):

$$
\begin{gathered}
x(t)=v\left(t-t_{1}\right)+\alpha v\left(t-t_{2}\right) \\
=v(t) *\left[\delta\left(t-t_{1}\right)+\alpha \delta\left(t-t_{2}\right)\right]
\end{gathered}
$$

where $\alpha$ is a proportional ratio of the second flat signal magnitude to the first one. Using Fourier transform of $x(t)$, the convolution of the time domain is transformed into an equation of the frequency domain, as shown in Equation (2). It is represented by Euler formula as both real values of the cosine function and imaginary values of the sine function with $1 / t_{1}$ and $1 / t_{2}$ cycles in the frequency domain:

$$
\begin{aligned}
& X(f)=\int_{-\infty}^{\infty} x(t) e^{-j w t} d t=V(f)\left[e^{-i w t_{1}}+\alpha e^{-i w t_{2}}\right] \\
& =\mathrm{V}(f)\left[\left(\cos \omega t_{1}+\alpha \cos \omega t_{2}\right)-i\left(\sin \omega t_{1}+\alpha \sin \omega t_{2}\right)\right]
\end{aligned}
$$

The frequency magnitude after Fourier transform is calculated as follows:

$$
\begin{gathered}
|X(f)|=|V(f)| \sqrt{1+\alpha^{2}+2 \alpha\left(\cos \omega t_{1} \cos \omega t_{2}+\sin \omega t_{1} \sin \omega t_{2}\right)} \\
=|V(f)| \sqrt{1+\alpha^{2}+2 \alpha \cos 2 \pi f\left(t_{2}-t_{1}\right)}
\end{gathered}
$$


where $\omega=2 \pi f$. The signal for two flats with time delays of $t_{1}$ and $t_{2}$ becomes a spectrum having a period of $1 /\left(t_{2}-t_{1}\right)$ in the frequency domain. Next, after taking the natural logarithm of its squared value, it is divided into the flat signal component and the impulseperiodic component as shown in Equation (4).

Here, by performing Fourier inverse transform, the cepstrum can be obtained as Equation (5), which is composed of a summation of a flat signal $C_{V}(\tau)$ and $C_{I}(\tau)$ impulse. The horizontal axis $\tau$ of the cepstrum has the same dimension to the time $t$, and is defined as Quefrency:

$$
\begin{aligned}
& \ln |X(f)|^{2}=\ln |V(f)|^{2}+\ln \left[1+\alpha^{2}+2 \alpha \cos 2 \pi f\left(t_{2}-t_{1}\right)\right] \\
& \ln |X(f)|^{2}=\ln |V(f)|^{2}+\ln \left[1+\alpha^{2}+2 \alpha \cos 2 \pi f\left(t_{2}-t_{1}\right)\right]
\end{aligned}
$$

Equations (6) and (7) show the spectral calculation results when wheel-flat signals are three or four, respectively. Assuming that the train running speed is constant, the time delay interval of the flat signals is equal to $t_{2}-t_{1}=t_{3}-t_{2}=t_{4}-t_{3}=T$. As the number of wheel-flat signals increases, the multiple components of the flat cycle $\mathrm{T}$ in the spectrum increase one by one:

$$
\begin{gathered}
\left|X_{3}(f)\right|=|V(f)| \sqrt{3+2(2 \cos \omega T+\cos 2 \omega T)} \\
\left|X_{4}(f)\right|=|V(f)| \sqrt{4+2(3 \cos \omega T+2 \cos 2 \omega T+\cos 3 \omega t)}
\end{gathered}
$$

Figure 1 shows the spectrums by periodic functions that are obtained converting four impulse signals to frequency of the flat cycle period $(T=0.243)$. The spectrums are composed of multiples of the flat cycle $\mathrm{T}$ as shown in Equation (7).

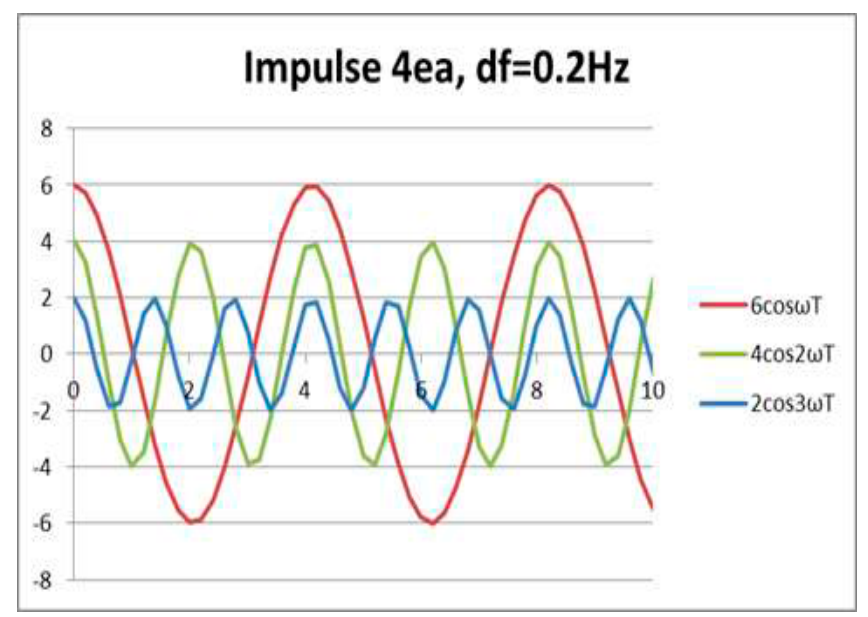

Figure 1. Period functions that form spectrums for four impulse signals.

The fundamental frequency in Figure 1 is $0.2 \mathrm{~Hz}$, and the magnitude of three cosine waveforms remains constant without attenuation. However, the magnitudes for waveforms are decreased and distorted with the frequency intervals shortened.

Because frequency intervals have a large effect on periodic components of the spectrum like the above example, the accuracy of cepstrum data becomes worse. To improve data resolution for high frequencies, a higher accuracy of spectrum and cepstrum data is necessary, and the time of data analysis gets longer. In addition, using zero padding prior to Fourier transform can be another helpful technique.

These domains in accordance with Equations (1)-(5) are shown in Figures 2-4. In the time domain of Equation (1), the periodic response signal of a flat is represented with a convolution of flat signal and impulse, as shown in Figure 2. In the frequency domain of 
Equation (3), Fourier transforms of the flat signal and the impulse signal are performed before the convolution, as in Figure 3. Then, by taking a logarithm of the convolution, it can be expressed as the sum of the flat signal and the periodic signal of the impulse. In the Quefrency domain of Equation (4), the analysis results are more affected by the impulse signal than the flat one in Figure 4.
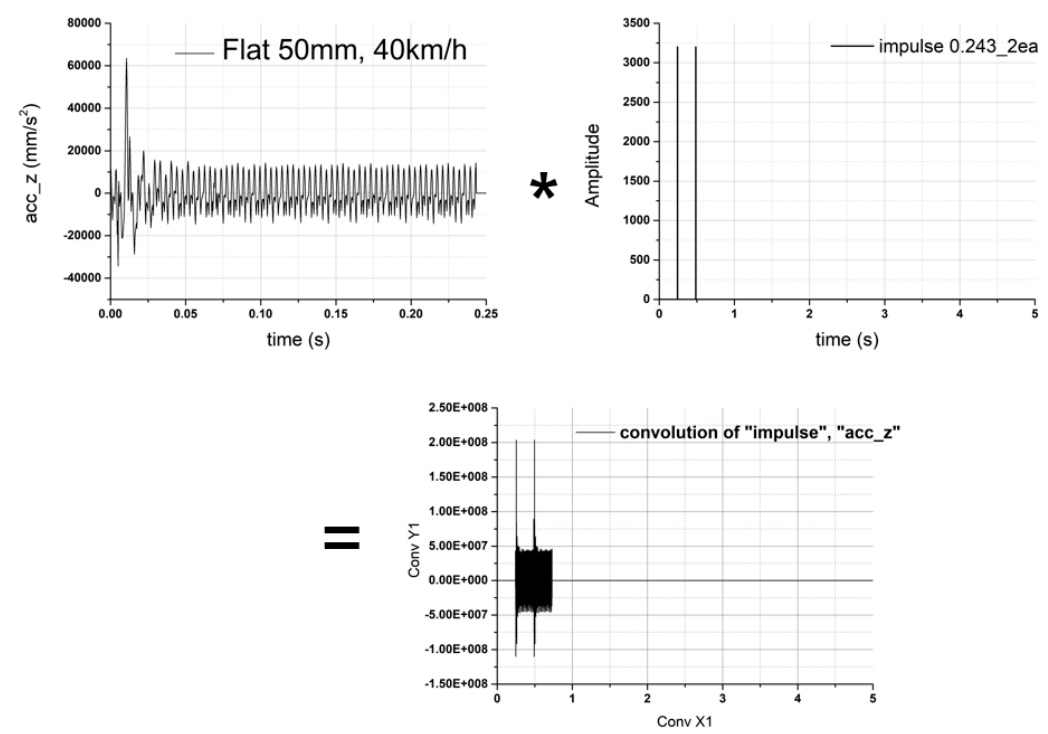

Figure 2. Time domain.
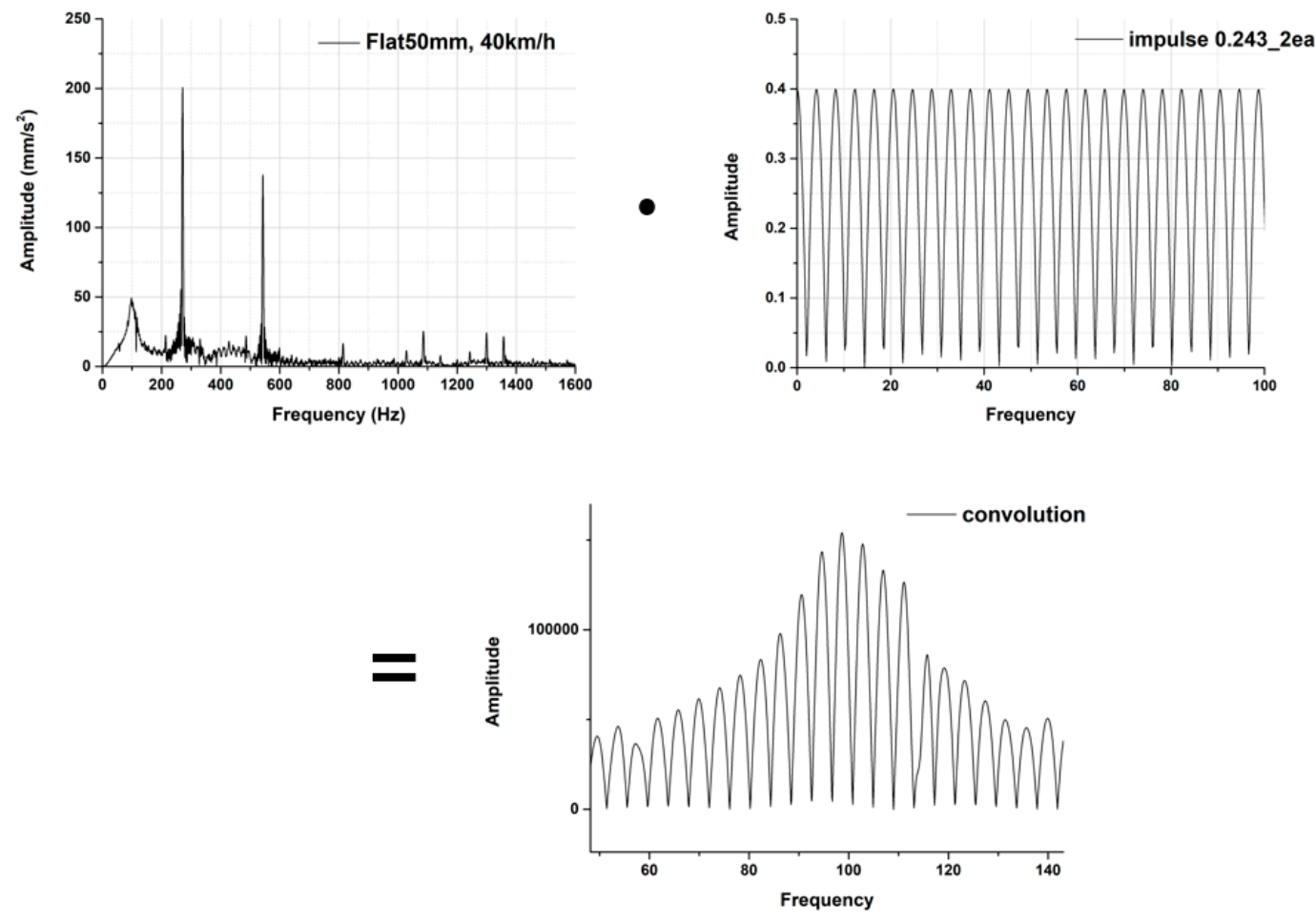

Figure 3. Frequency domain. 

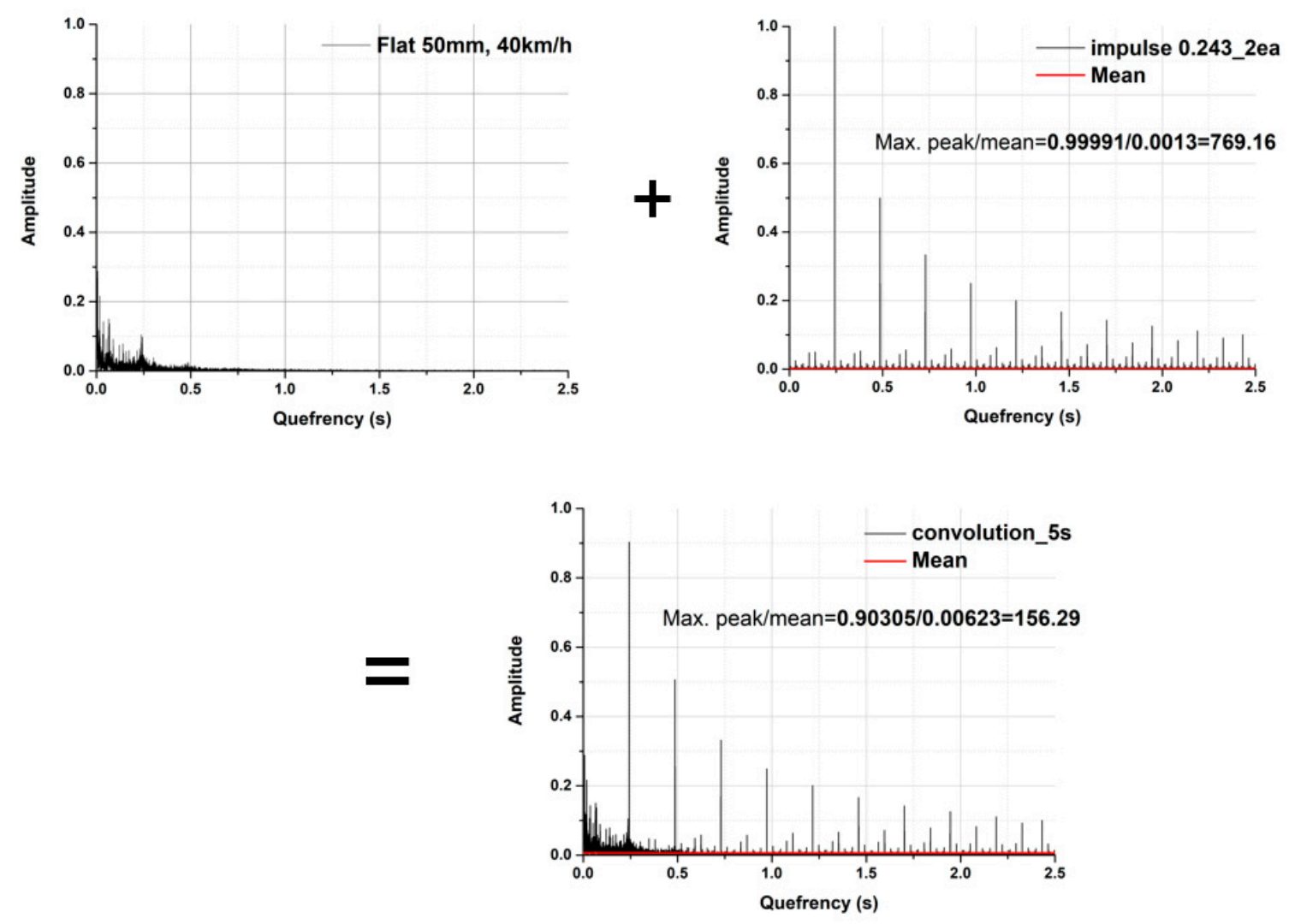

Figure 4. Separation of the wheel flat signal and the impulse signal in the Time, Frequency, and Quefrency domains.

Summarizing this section, the cepstrum is an effective signal processing technique to detect only the cyclic signal characteristics, which is less affected by the size or the width of wheel flats.

\subsection{Simulation Modeling of a Wheel Flat}

To analyze the vibration signal of a defected wheel with flats, dynamic simulations were performed using commercial software, Recurdyn. Figure 5 is a bogie model composed of a bogie frame, suspensions, and rails for the Seoul urban railway line 7. Figure 6 is the defected wheel model with a flat. The vibration signal was extracted from the axle box of the flat wheel.

\subsection{Cepstrum Analysis Technique under a Variable Velocity Condition}

The cepstrum is useful to detect periodic impulse signals at a constant speed. However, the cycle of vibration signal with a flat gets changed under variable velocity conditions of trains. This problem can be solved by an order analysis changing the time domain signal of vibration extracted from the axle box into the rotation angle domain signal of the wheel axis. In the case of railway vehicles, speed sensors and tachometers are always attached to the axle boxes to measure the rotational speed of axles, so no additional sensor is required for this domain change.

Fourier transform based on the time domain would not be proper because the angular time intervals are not uniform at varying train speed unless the time domain is changed to the wheel rotation angle domain. For the order analysis, after exchanging the domains, interpolation must be applied to equalize the uneven angular intervals caused by the varying speed. Figure 7 is a block diagram of the current analysis that existing cepstrum analysis is combined and order analysis to effectively detect flats under operating conditions with variable speed. 


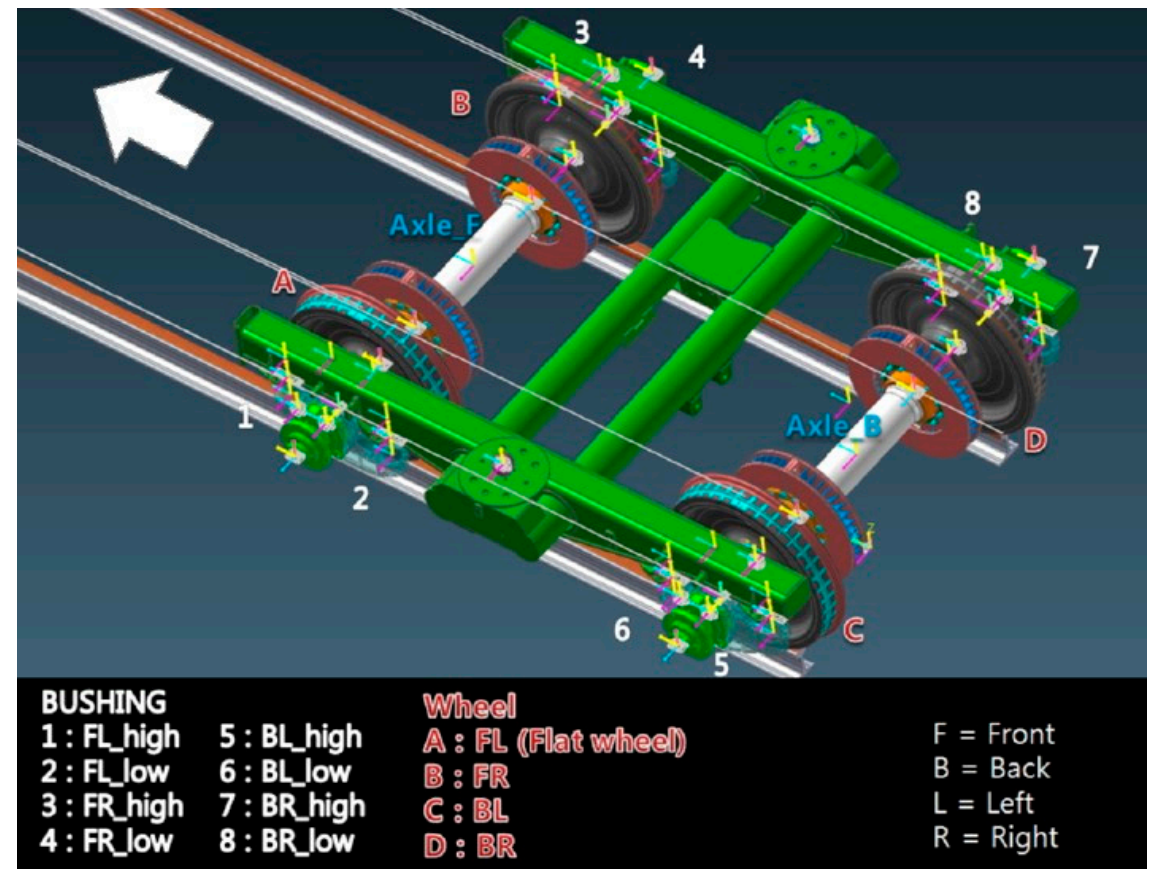

Figure 5. Bogie modeling.

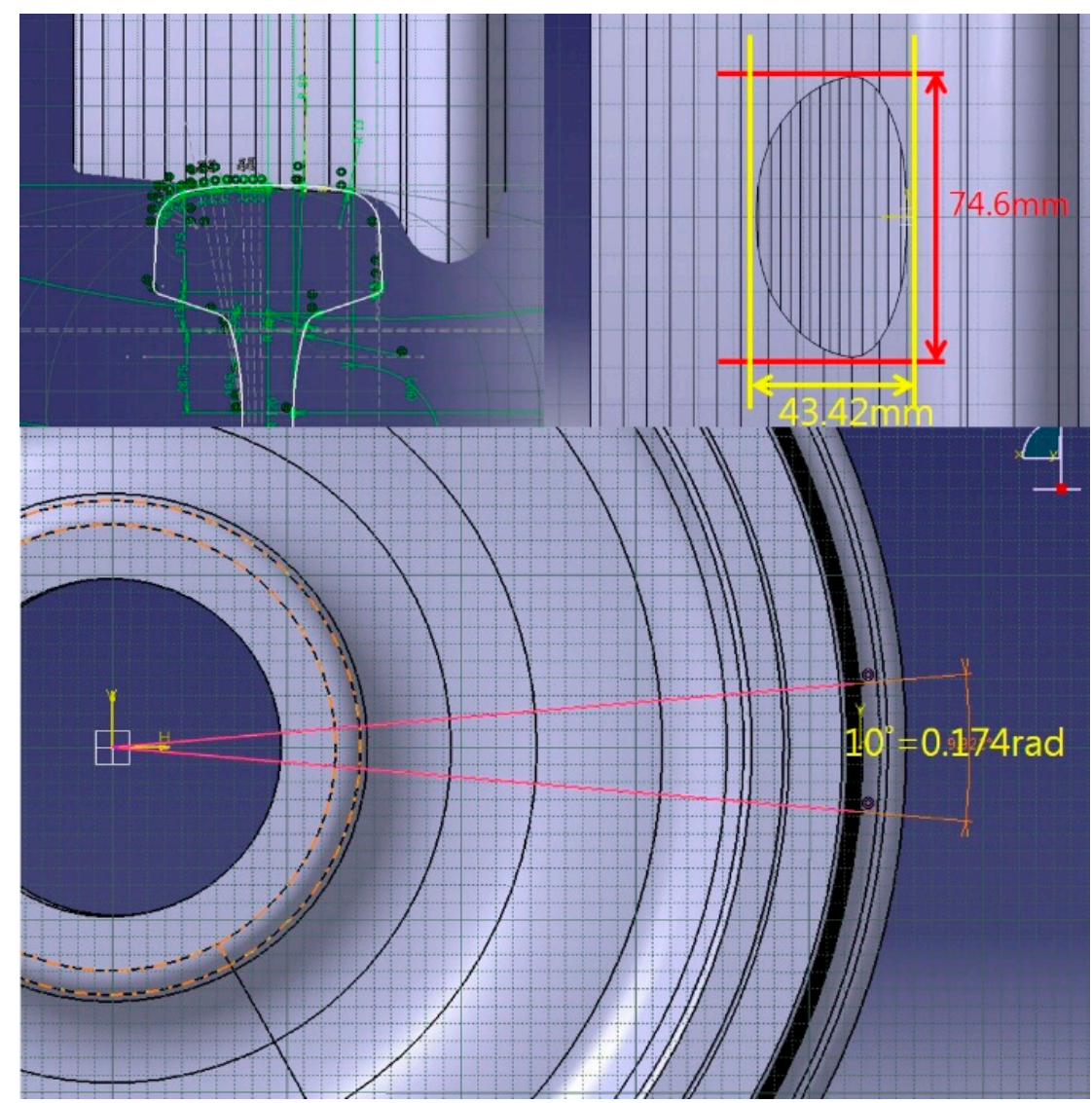

Figure 6. Flat wheel modeling. 


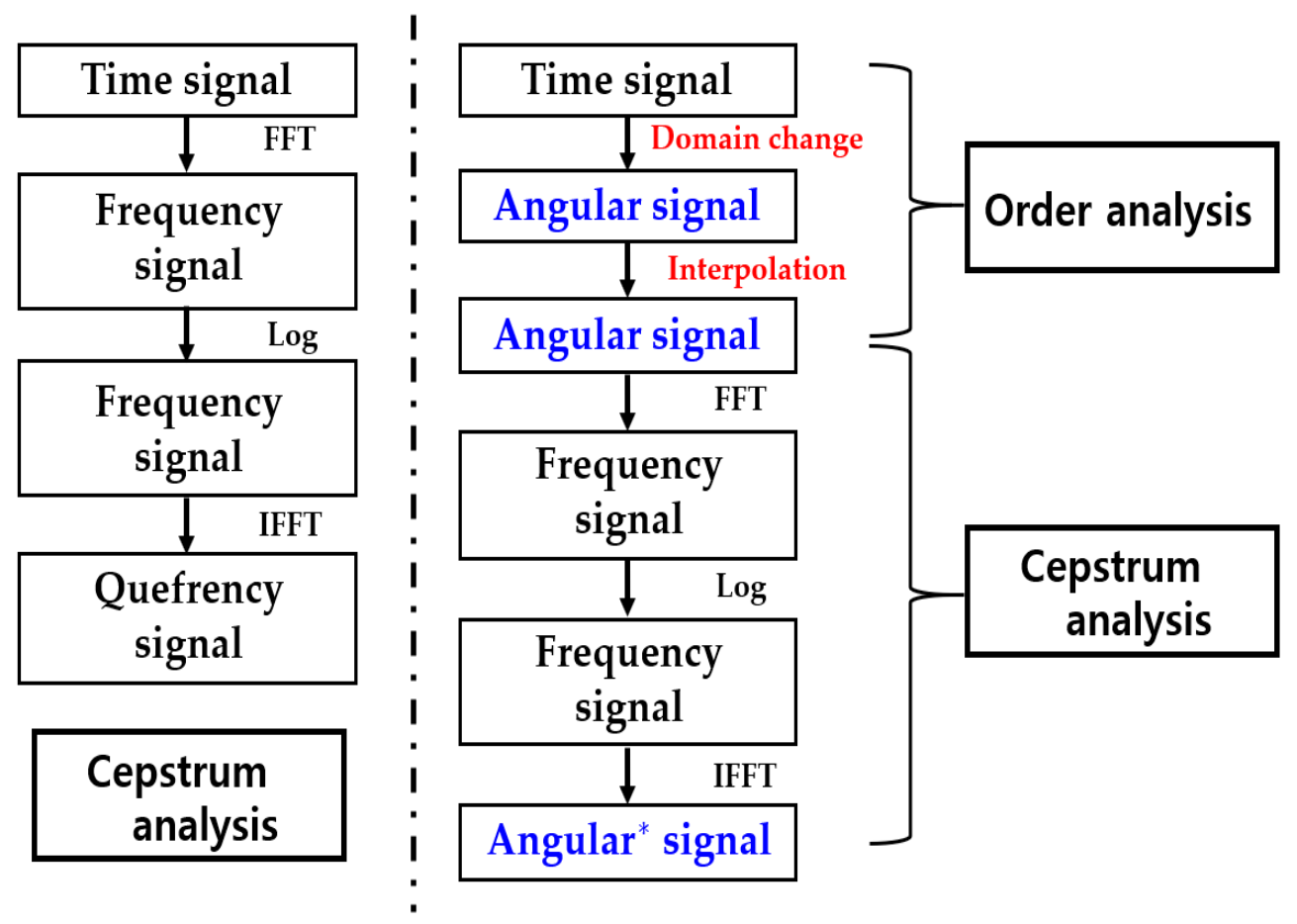

Figure 7. The existing cepstrum analysis vs. the current cepstrum analysis combined with the order analysis.

Figure 8 is a time domain signal extracted from simulation of wheel-rail dynamics at $2 \mathrm{~km} / \mathrm{h} / \mathrm{s}$ acceleration, and the speed was increased from $40 \mathrm{~km} / \mathrm{h}$ to $50 \mathrm{~km} / \mathrm{h}$ for 5 seconds. As shown in Figures 9 and 10, the time domain was changed into the rotation angle domain of the wheel axis, and the angular intervals were equalized using interpolation of signal data points. After that, the cepstrum was applied, and the flat signal detection was obtained as shown in Figure 11.

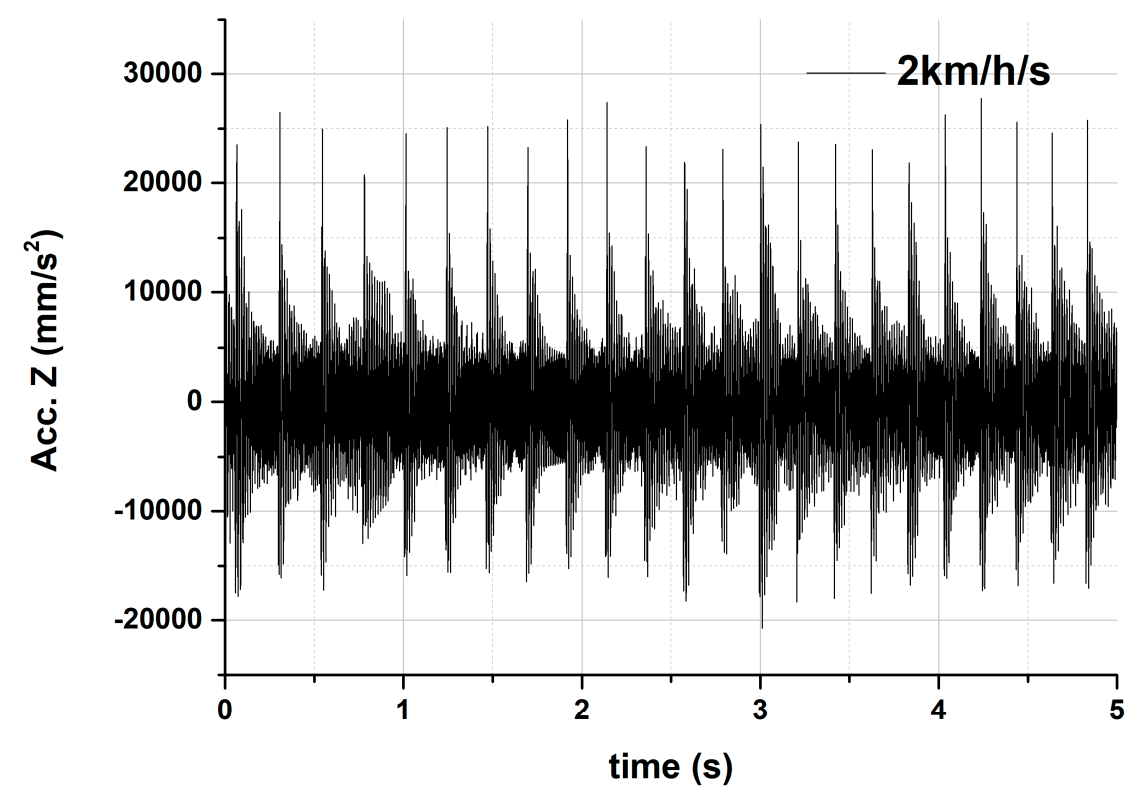

Figure 8. Time domain signal of a wheel flat. 


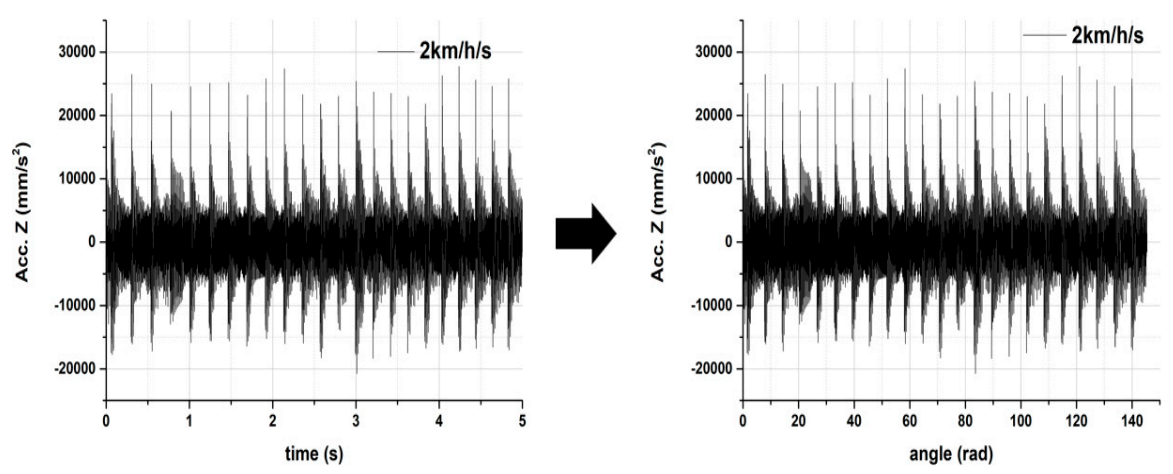

Figure 9. Change of the time domain into the rotation angle domain.
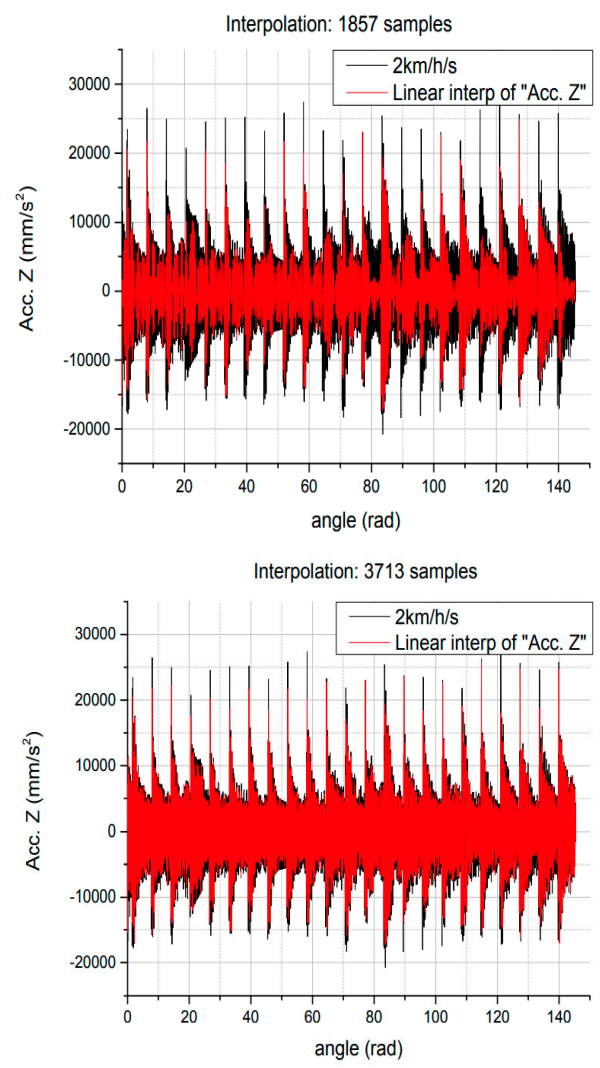

(a) Using $25 \%, 50 \%$ interpolation data samples.

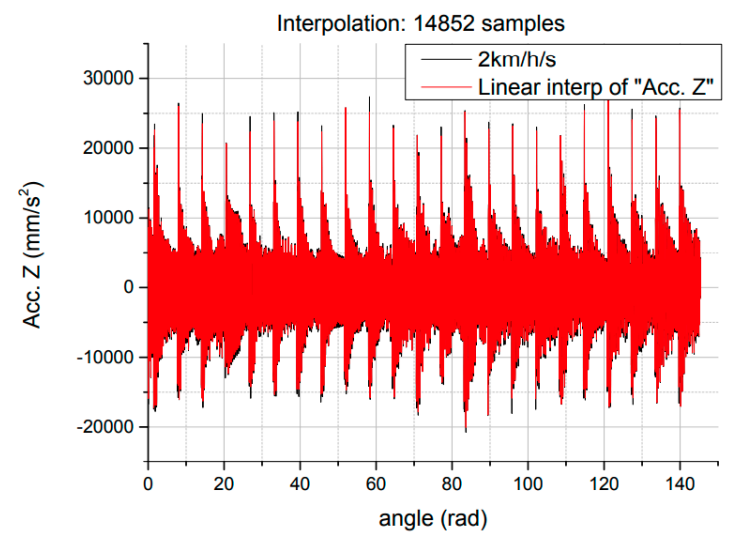

(b) Using $200 \%$ interpolation data samples.

Figure 10. The conformability of the original data according to the number of data samples. 


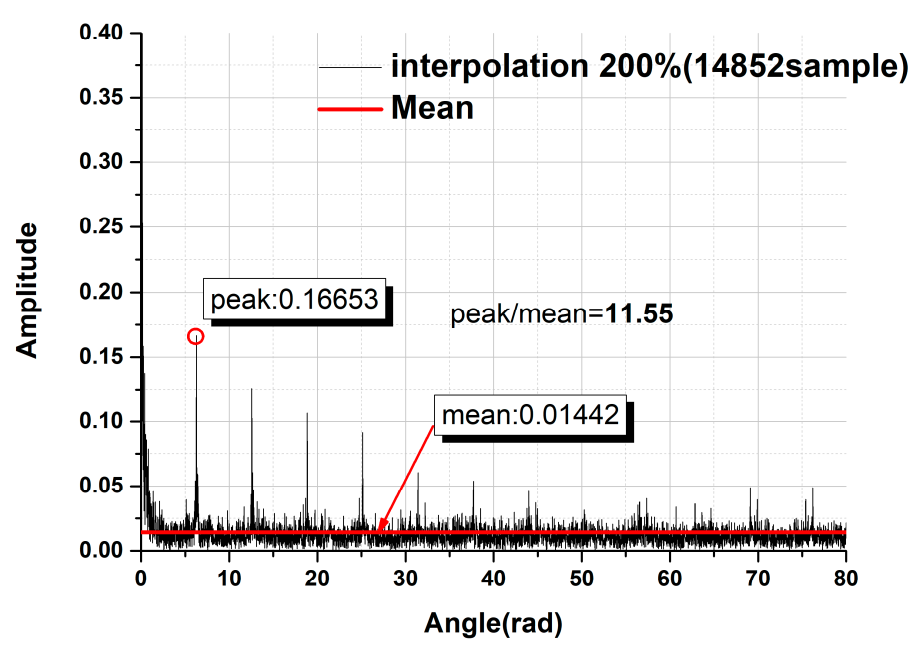

Figure 11. Cepstrum results of a single flat in the angle domain.

By the way, as the angle data intervals become narrower at the interpolation step, the original signal of the flat can be accurately recovered, and the frequency resolution is also improved. In addition, the flat cyclic signal component at every $2 \pi$ and the peak/mean value tends to increase. Figure 10 shows that the conformability to the original data is improved as the number of data samples increases using narrower angle data intervals.

\subsection{Detecting the Harmonic Component of Flat Signals from the Vibration Signal Including Noise Signals}

In order to verify that the cepstrum analysis is effective in the case including noise signals by the rail irregularities and the vibrations of bogie components, such as axle bearings and reduction gears, the vibration signals of a normal wheel were measured using acceleration sensors during operation of the Seoul subway Line 7, as shown in Figure 12. The non-contact type speed sensor that generates 90 pulses per a revolution of the wheel was innately installed in the bogie. Using this speed sensor signal, the measured vibration signal can be converted into an angular domain signal. The measured data is shown in Figure 13.

The vibration signal of the flat wheel was obtained superimposing the simulation result at the same speed condition on the measured vibration signal of the normal wheel, and then it was analyzed by the cepstrum.

The cepstrum analysis results are shown in Figure 14. The peak factor of the flat wheel is approximately two times that of the normal wheel including only the noise signal. The peak value is detected in the harmonic components of the wheel rotation. The flat signal was detected by the cepstrum analysis, even though the flat signal is not visible in the vibration signal of the flat wheel because the magnitude of the noise signal is about four times that of the flat signal.

\subsection{Analysis of Data Tested by Roller Rig}

In the actual operation, passengers ride on in service. Thus, it is difficult to conduct measurement tests using a wheel with flats. Therefore, the vibration test was conducted using a roller rig in the Korea Railroad Research Institute.

The measurement test using a roller rig is shown in Figure 15. The wheel test conditions are $20 \mathrm{~km} / \mathrm{h}$ in uniform velocity and a flat of $50 \mathrm{~mm}$ at the left wheel. In addition, the data were obtained by installing a three-axis accelerometer in the axle box. The tests were conducted more than three times to obtain good data. The test results are shown in Figure 16. Figure 16a shows all the acceleration signals measured in the two 3-axis accelerometers. The flat signals were measured even on the right wheel without a flat. However, the largest vibration signal was measured on the left wheel z-axis, which is most related to the flat. 


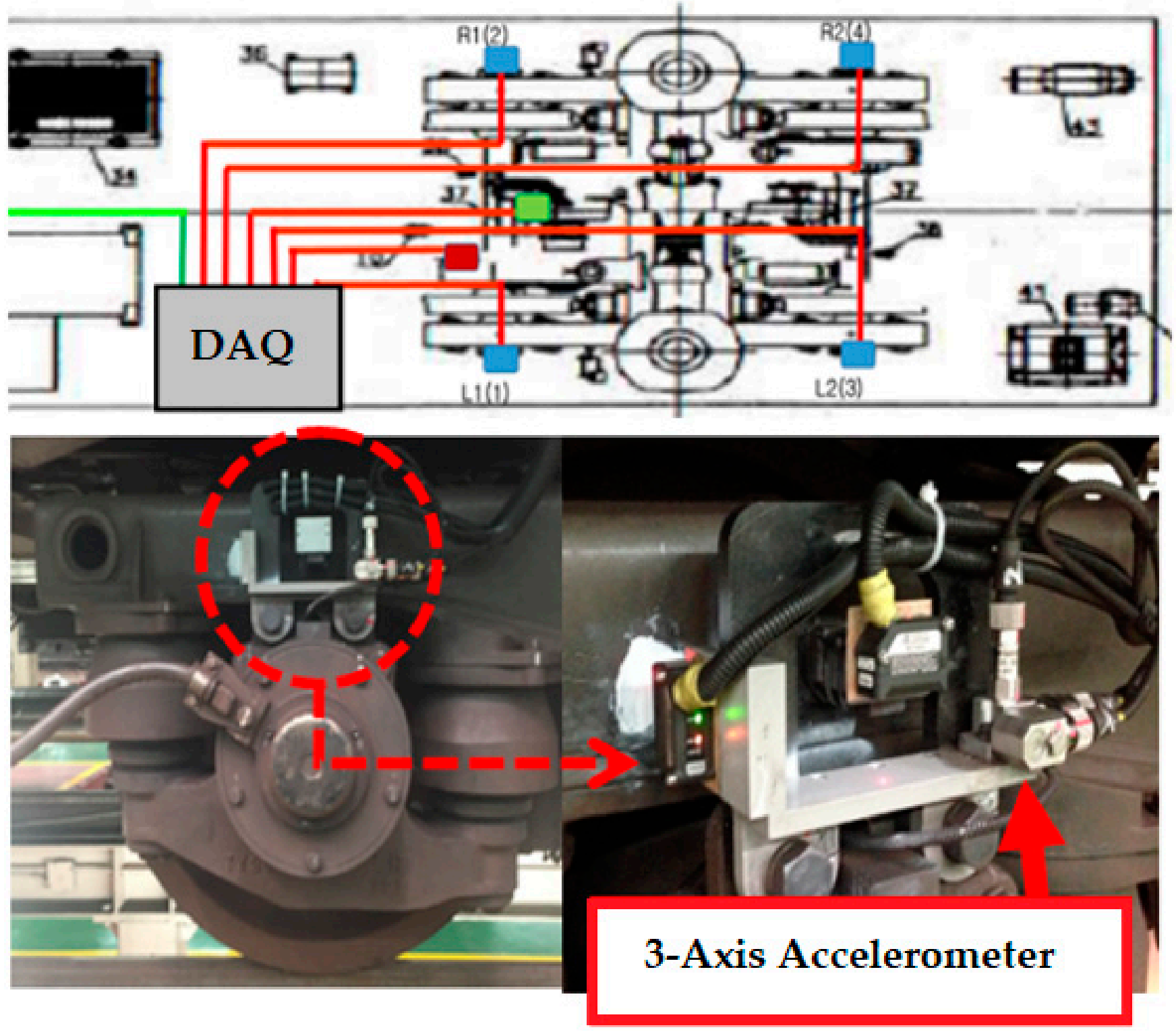

Figure 12. Acceleration sensor installation.

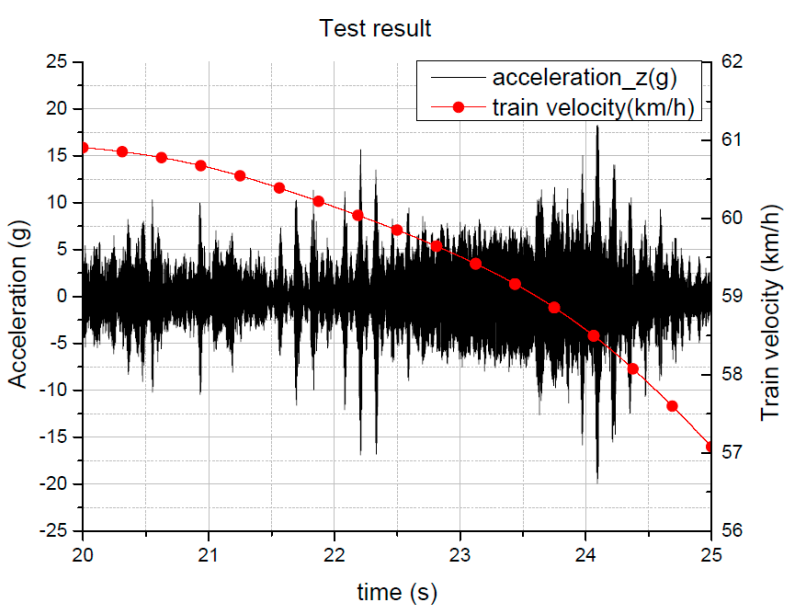

Figure 13. The test measurement result for a high irregularity section of a rail. 

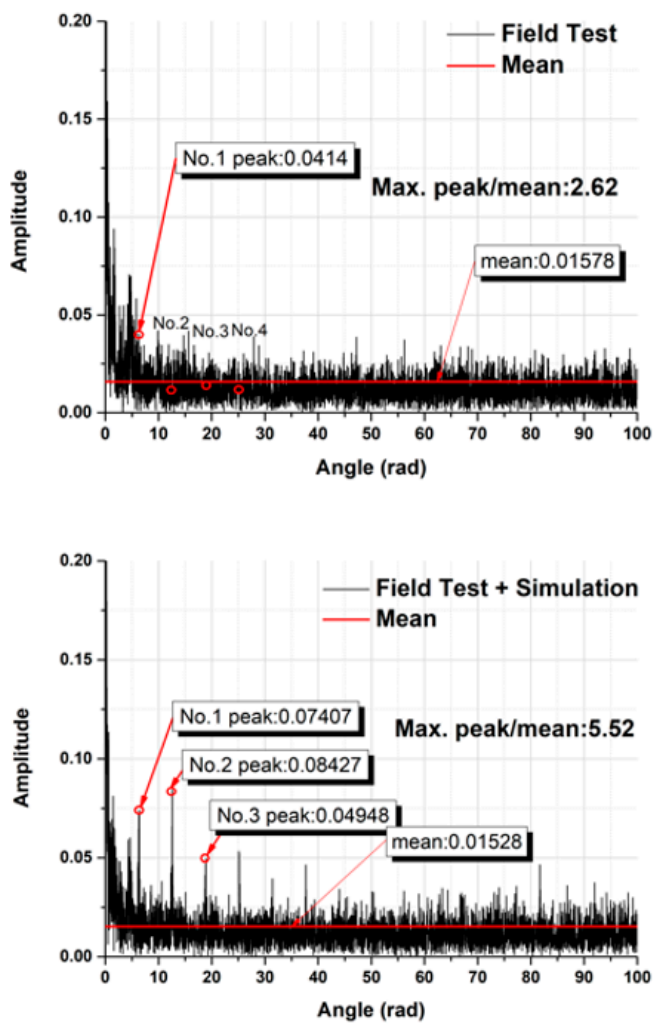

Figure 14. Cepstrum results depending on the absence (the upper) or presence (the below) of a wheel flat.

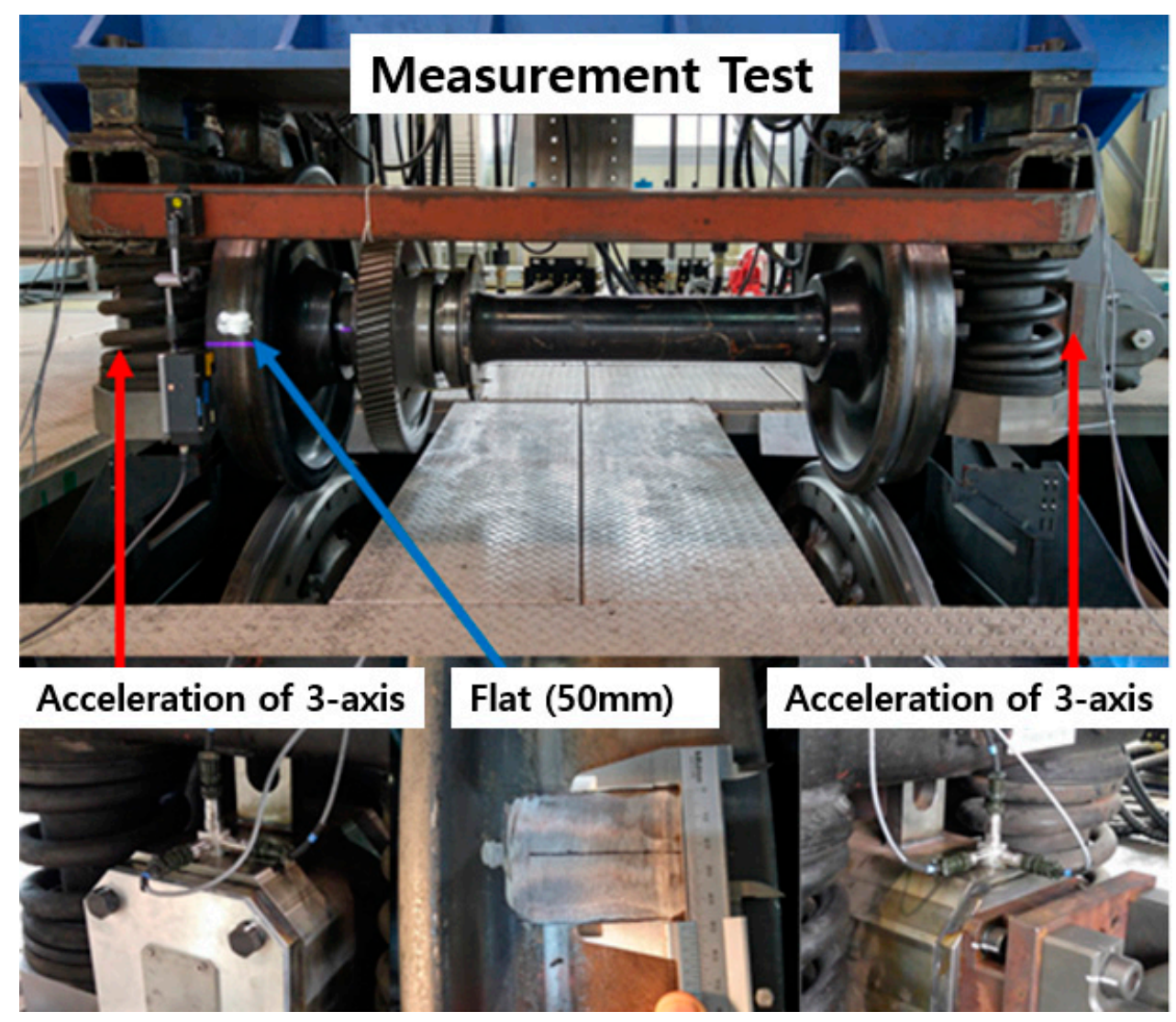

Figure 15. Measurement test using a roller rig (wheel with a $50 \mathrm{~mm}$ flat). 

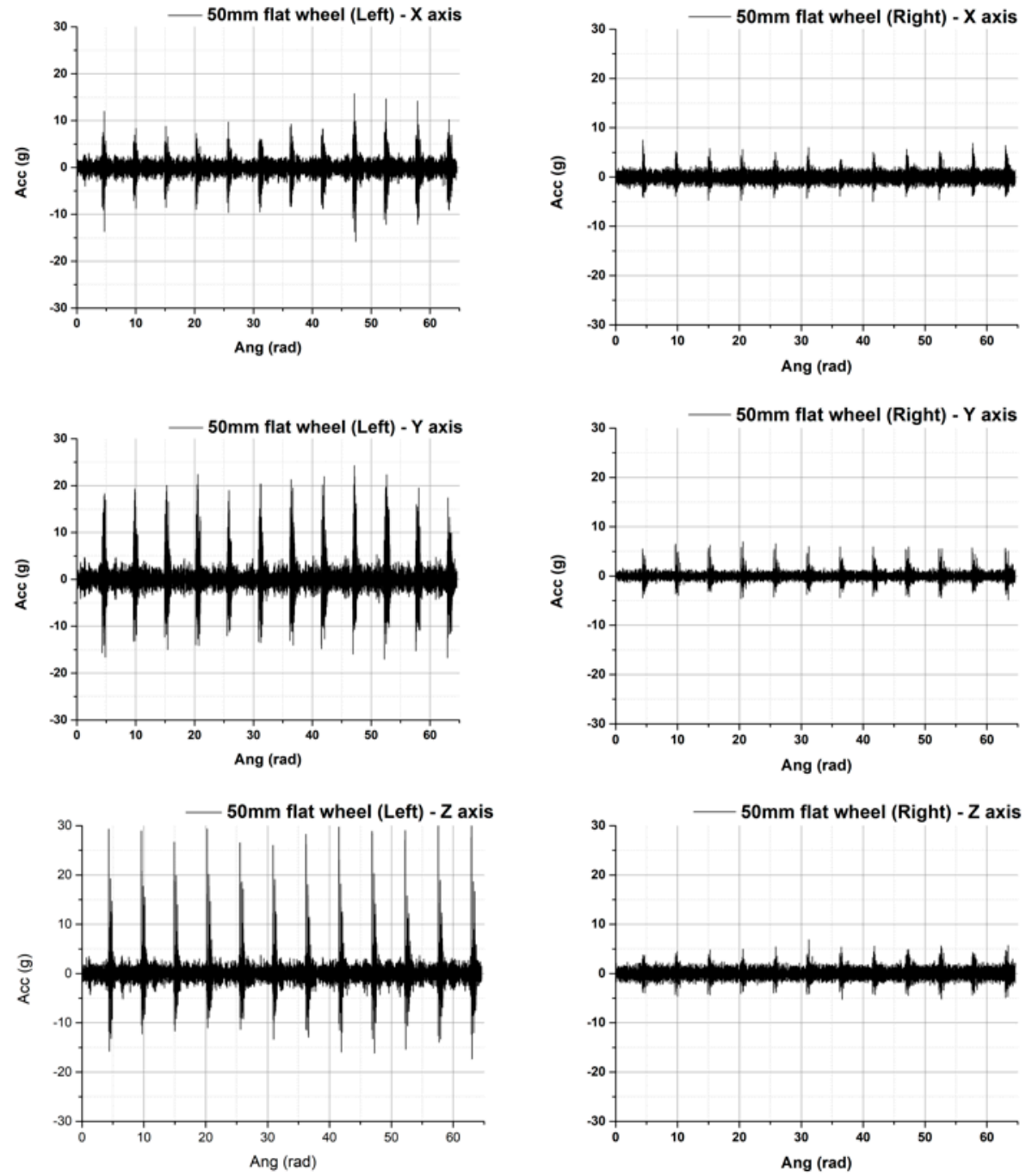

(a) Acceleration signals measured in the two 3-axis accelerometers.

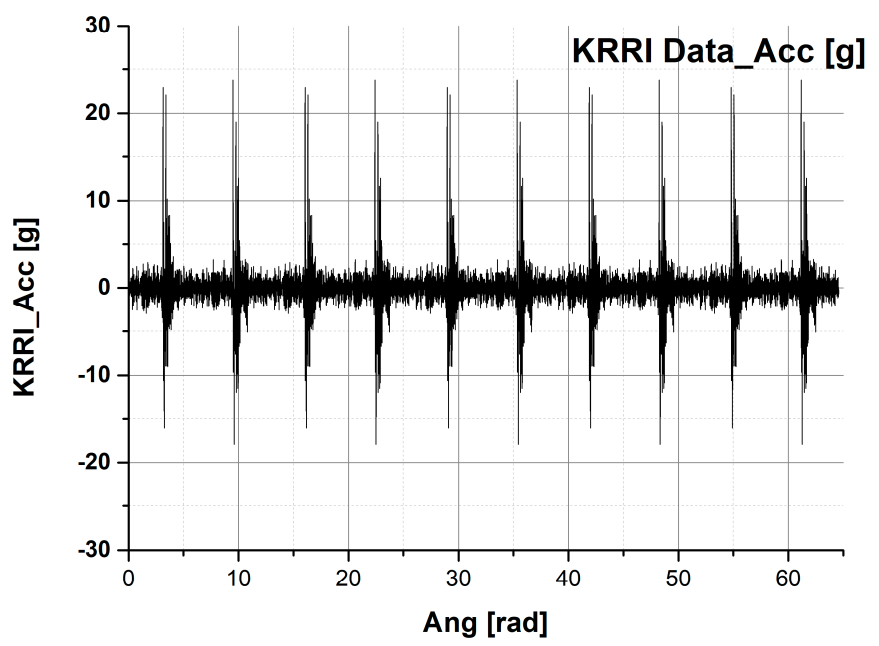

(b) z-axis acceleration of the left wheel.

Figure 16. Vibration signals on the $50 \mathrm{~mm}$ flat wheel at a speed of $20 \mathrm{~km} / \mathrm{h}$. 
Figure $16 \mathrm{~b}$ is the $\mathrm{z}$-axis acceleration of the left wheel. A periodic harmonic component of about $20 \mathrm{~g}$ occurred at vertical (z-axis) acceleration.

Figure 17 shows the results of a cepstrum analysis using the flat signal shown in Figure 16b. As a result of the cepstrum analysis, it was confirmed that peak values are occurred in the harmonic components. Therefore, based on the results of Figure 17, it is confirmed that the cepstrum analysis method is appropriate for not only simulation but also measurement data.

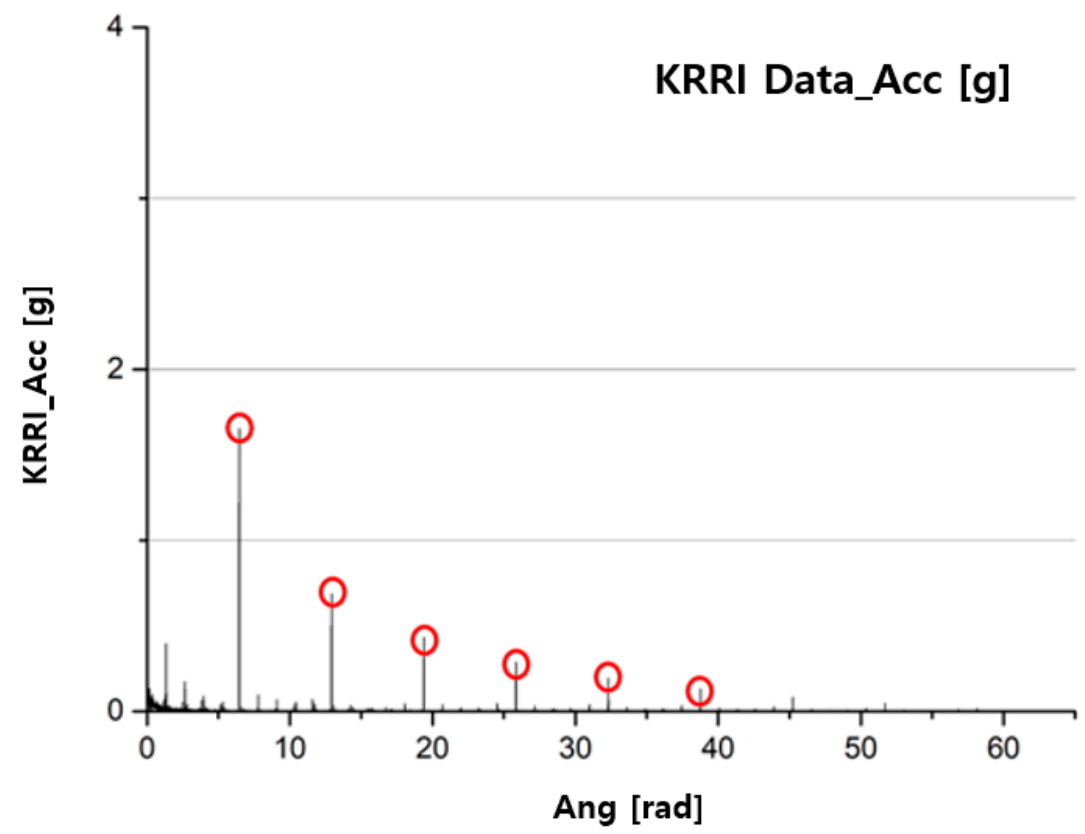

Figure 17. Results of the cepstrum analysis using the testing data of the roller rig.

\section{Cross Correlation Analysis to Diagnose the Sizes of Wheel Flats}

\subsection{Definition of Cross Correlation}

The cepstrum analysis is effective in detecting the existence of flats, but not their sizes. Therefore, another signal-processing technique must be applied to diagnose the sizes of wheel flats.

The magnitude and width of a waveform signal depend on the size of a flat and the speed of a vehicle [17]. Cross correlation analysis is one of the signal processing techniques that effectively consider the signal waveform area of a wheel flat. As shown in Equation (8), this technique is to calculate the overlapping area of the kernel signal (g) with the measurement signal (f), while kernel signal (g) moves along with the time axis. In the case of a discrete function rather than a continuous function, the equation is given as in Equation (9). [18]:

$$
\begin{gathered}
(f \star g)(t)=\int_{-\infty}^{\infty} f(\tau) g(t+\tau) d \tau \\
(f \star g)(n)=\sum_{-\infty}^{\infty} f[m] g[m+n]
\end{gathered}
$$

In the cross correlation analysis, the maximum peak value can be obtained using the same kernel signal as the measurement signal. In this paper, we used the flat signal of the simulation result as the kernel signal.

\subsection{Diagnosis of a Flat Size from Flat Signals Including Severe Field Noises}

The normal wheel signal including severe field noises was obtained from a field vehicle test under operating conditions with variable speed, as shown in Figure 13. By the way, the wheel flat signal was obtained by test in the KRRI (Korea Railroad Research Institute). 
Then, a final flat signal including severe field noises was obtained by superimposing the above two signals which are the field operation signal on a normal wheel and the tested one on a wheel flat.

Subsequently, cross correlation analyses with kernel function $(\mathrm{g})$ for the final flat signal, combined with the order analysis, were performed, as shown in Figure 18. It is found in Figure 18 that the cross correlation peaks using the one flat signal as a kernel function are significantly amplified compared to the noise signal peaks. However, it is somewhat difficult to distinguish the flat signal peaks from the noise signal ones because the peak magnitude of the amplified flat signal is a little similar to the noise ones.
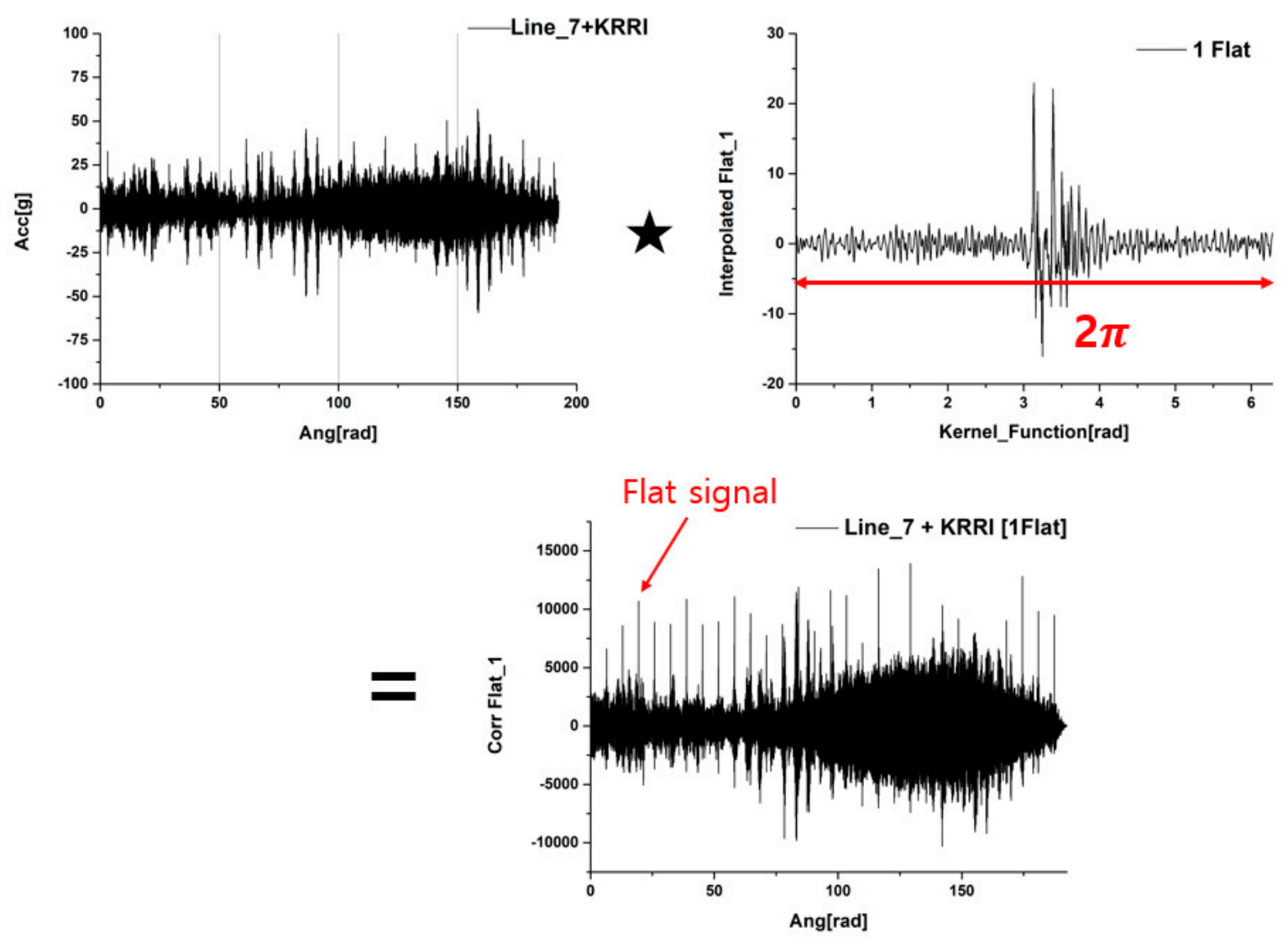

Figure 18. Cross correlation result of the flat wheel using one cycle signal for one flat.

In Figure 19, four flat signals were selected as kernel function g. After that, the cross correlation analysis was performed again. In this analysis, a flat signal was selected as the representative kernel function $g$ for four flat signals of $2 \pi$ cycle, using the characteristic that the cycle of the flat signal is constant as $2 \pi$ rad in the angle domain.

The cross correlation result of peaks on the four-flat signals is about two times larger than that of the noise signal. As shown in Figures 18 and 19, the cross correlation result using the kernel function (g) with the four flat signals makes the flat signal more clearly visible compared to the cross correlation result with one flat signal. As the number of the flat signals used in the g-function increases, the amplification rate of the flat signal also increases. Moreover, the cross correlation result of the noise signal is not amplified regardless of the number of the flat signals used in the g-function.

Figure 20 shows the results of the cepstrum and cross correlation analyses for the six different flat sizes which are normalized by the analysis result of the flat size of $10 \mathrm{~mm}$. When comparing the analysis results of the cepstrum to those of the correlation, the cross correlation results indicate a linearly increasing trend according to the flat sizes. It means that the cross correlation is more effective than the cepstrum in diagnosing wheel flat sizes. 

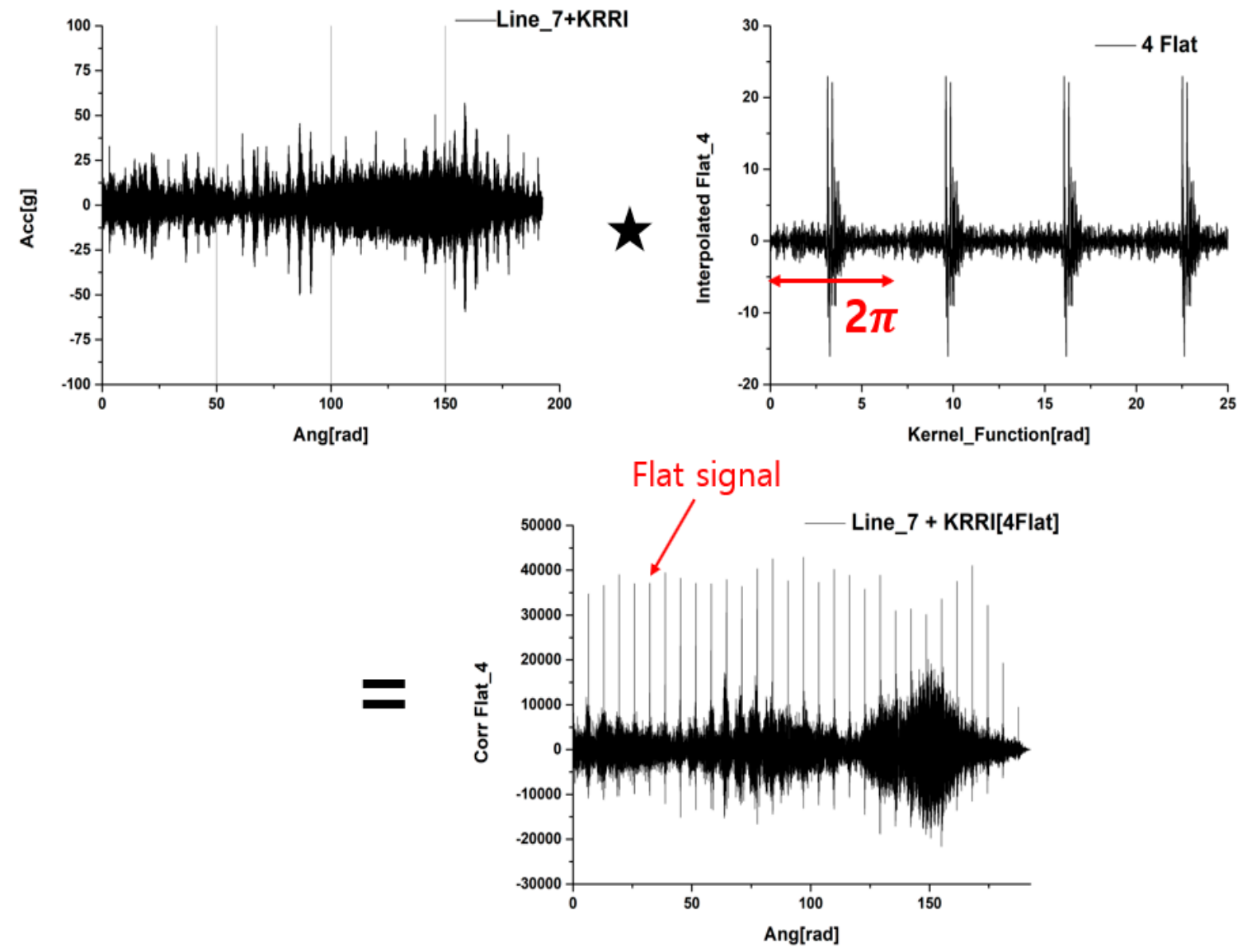

Figure 19. Cross correlation result of the flat wheel using four cycles signal for one flat.

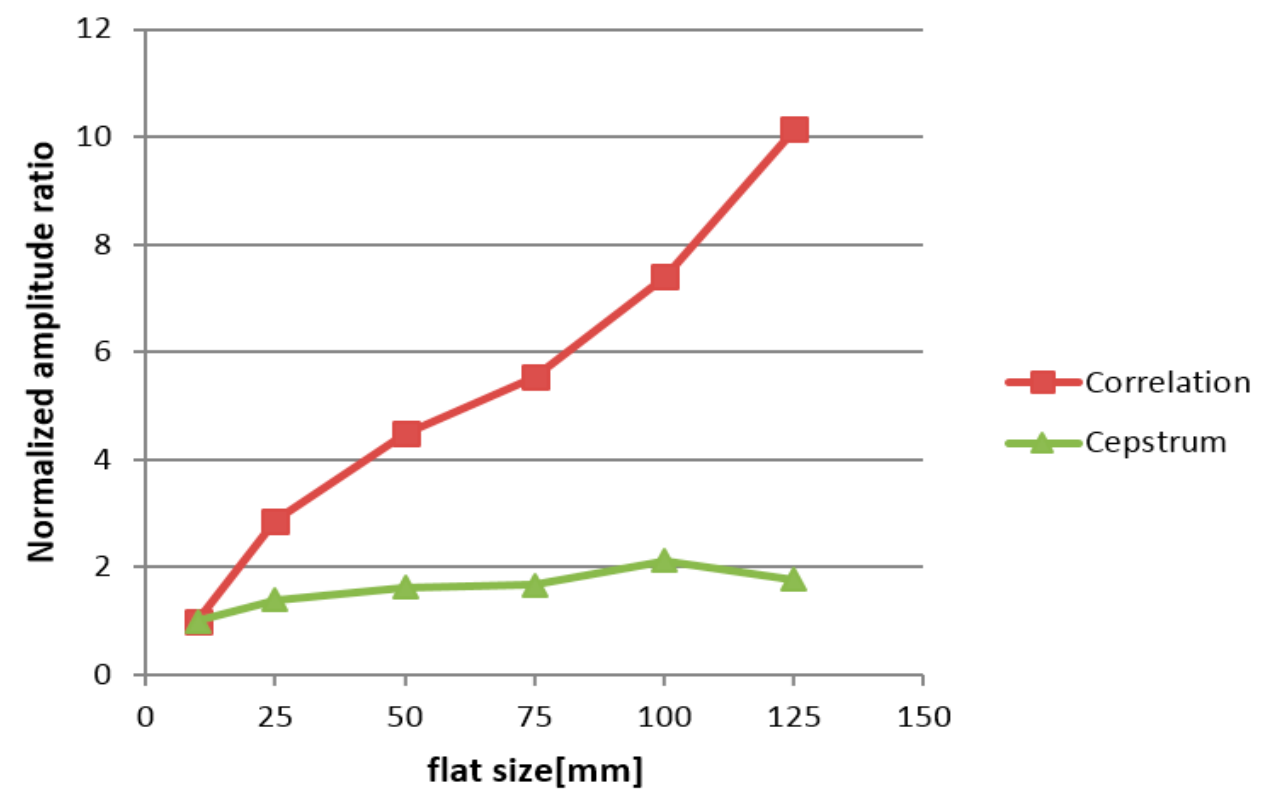

Figure 20. Normalized amplitude ratios according to flat sizes.

\section{Conclusions}

This study suggested two signal processing methods to detect wheel flats and diagnose flat sizes. The first one is cepstrum analysis combined with order analysis to consider variable speed conditions. The order analysis converts the time domain into a rotating 
angular domain. The second one is cross correlation analysis combined with the order analysis for detecting flat signals in severe noise signals.

The cepstrum analysis is effective in detecting wheel flats. In particular, the cepstrum analysis combined with the order analysis is suitable for application to railway vehicles because speed sensors and tachometers are already installed to measure the rotational speed of the wheels. In order to increase the detectability, the following measures can be summarized: First, use the zero padding to improve the frequency resolution. Second, use the greater amount of sampling data when interpolating in the rotation angle domain; then, it can improve the frequency resolution. In this case, an interpolation using compact data points enough to cover the peak value of the vibration signal must be performed to obtain the equal angular intervals after changing the domains. Otherwise, the sensitivity of the flat signals in the cepstrum analysis is significantly deteriorated.

As mentioned above, the cepstrum analysis is very useful to detect the periodic signal of wheel flats, but there is a limit to diagnose wheel flat sizes. However, this can be supplemented using the cross correlation analysis. The procedure to diagnose wheel flat sizes using the correlation analysis is as follows: first, flat signals should be changed to the angle domain from the time domain. By doing so, the cross correlation analysis result can be by greatly improved because it is well matched to the kernel function of the wheel flat signal even under variable speed conditions. Second, use the cross correlation kernel function with the uniformity of the wheel flat signal in the angle domain. Several flat signals can be used to amplify the cross correlation flat signals in proportion to its number of each $2 \pi$ angle. The flat signals amplified by the cross correlation analysis will become several times larger than the severe noise signals. Therefore, the advantage of the cross correction analysis is that it can detect flat signals even in severe noise signals. However, the disadvantage is that kernel signals are necessary to apply it. Therefore, to solve for these disadvantages, flat signals should be built in the database when flats occur. If the database is established, the flat size can be diagnosed through the cross correlation analysis. In addition, it is also possible to figure out the flat numbers by counting the amplified flat signals generated during one revolution of the wheel.

In the near future, the proposed algorithms on the flat signal processing will be provided to railway companies so that they can diagnose the actual faults after inputting them into the equipment using Python code. If it happens, for an actual operation and maintenance, the proposed algorithm can be appropriately applied to measurement, analysis, and diagnosis process of wheel flats in real time.

Author Contributions: Conceptualization, J.S., G.K. and J.K.; methodology J.S., G.K. and J.K.; software J.S. and B.C.; validation J.S., G.K. and J.K.; writing—review and editing, J.S., B.C. and J.K.; supervision, J.K.; project administration, J.K.; funding acquisition, Seoul National University of Science and Technology. All authors have read and agreed to the published version of the manuscript.

Funding: This study was supported by the Research Program funded by SeoulTech (Seoul National University of Science and Technology).

Conflicts of Interest: The authors declare no conflict of interest.

\section{References}

1. Jardine, A.K.; Lin, D.; Banjevic, D. A review on machinery diagnostics and prognostics implementing condition-based maintenance. Mech. Syst. Signal Process. 2006, 20, 1483-1510. [CrossRef]

2. Du, W.-T.; Zeng, Q.; Shao, Y.-M.; Wang, L.-M.; Ding, X.-X. Multi-Scale Demodulation for Fault Diagnosis Based on a WeightedEMD De-Noising Technique and Time-Frequency Envelope Analysis. Appl. Sci. 2020, 10, 7796. [CrossRef]

3. Chen, S.Q.; Du, M.G.; Peng, Z.K.; Feng, Z.P.; Zhang, W.M. Fault diagnosis of planetary gearbox under variable-speed condi-tions using an improved adaptive chirp mode decomposition. J. Sound Vib. 2020, 468, 115065. [CrossRef]

4. Zhang, X.; Zhu, J.; Wu, Y.; Zhen, D.; Zhang, M. Feature Extraction for Bearing Fault Detection Using Wavelet Packet Energy and Fast Kurtogram Analysis. Appl. Sci. 2020, 10, 7715. [CrossRef]

5. Lu, K.; Wu, N.; Zhang, K.; Fu, C.; Jin, Y.; Yang, Y.; Zhang, H. Dynamical Behaviors Analysis of the Rotor Model with Coupling Faults and Applications of the TPOD Method. Appl. Sci. 2020, 10, 7415. [CrossRef] 
6. Duan, Y.; Wang, C.; Chen, Y.; Liu, P. Improving the Accuracy of Fault Frequency by Means of Local Mean Decomposition and Ratio Correction Method for Rolling Bearing Failure. Appl. Sci. 2019, 9, 1888. [CrossRef]

7. Zheng, Z.; Song, D.; Xu, X.; Lei, L. A fault diagnosis method of bogie axle box bearing based on spectrum whitening de-modulation. Sensors 2020, 20, 7155. [CrossRef] [PubMed]

8. Glowacz, A. Fault diagnosis of electric impact drills using thermal imaging. Measurement 2021, 171, 108815. [CrossRef]

9. Liang, B.; Iwnicki, S.; Feng, G.; Ball, A.; Tran, V.T.; Cattley, R. Railway wheel flat and rail sur-face defect detection by time-frequency analysis. Chem. Eng. Trans. 2013, 33, 745-750.

10. Gao, R.; He, Q.; Feng, Q. Railway Wheel Flat Detection System Based on a Parallelogram Mechanism. Sensors 2019, 19 , 3614. [CrossRef] [PubMed]

11. Choi, Y.-C.; Kim, Y.-H. Fault detection in a ball bearing system using minimum variance cepstrum. Meas. Sci. Technol. 2007, 18, 1433-1440. [CrossRef]

12. Gao, R.; He, Q.; Feng, Q.; Cui, J. In-Service Detection and Quantification of Railway Wheel Flat by the Reflective Optical Posi-tion Sensor. Sensors 2020, 20, 4969. [CrossRef] [PubMed]

13. Bogert, B.P. The quefrency analysis of time series for echoes: Cepstrum, pseudo-autocovariance, cross-cepstrum and saphe cracking. Time Ser. Anal. 1963, 209-243.

14. Gárdonyi, G.; Samu, K. Hybrid spectral speed determination method for rotary machines. J. Vibroeng. 2019, 21, 538-555. [CrossRef]

15. Bhakta, K.; Sikder, N.; Nahid, A.A.; Islam, M.M.M. Fault Diagnosis of Induction Motor Bearing Using Cepstrum-based Preprocessing and Ensemble Learning Algorithm. In Proceedings of the 2019 International Conference on Electrical, Computer and Communication Engineering (ECCE), Cox's Bazar, Bangladesh, 7-9 February 2019; p. 18566124.

16. Randall, R.; Antoni, J.; Smith, W. A survey of the application of the cepstrum to structural modal analysis. Mech. Syst. Signal Process. 2019, 118, 716-741. [CrossRef]

17. Time-frequency analysis of wheel-rail shock in the presence of wheel flat. J. Traffic Transp. Eng. 2014, 6, 457-466.

18. Yarlagadda, R.K.R. Analog and Digital Signals and Systems; Springer: Berlin, Germany, 2010. [CrossRef] 